\title{
Effects of antibiotic treatment on the fecundity of Rhipicephalus haemaphysaloides ticks
}

\author{
Lan-Hua Li ${ }^{1,2}$, Yi Zhang $^{2}$ and Dan Zhu ${ }^{2 *}$
}

\begin{abstract}
Background: Endosymbiotic bacteria inhabit a variety of arthropods including ticks and may have multiple effects on the host's survival, reproduction or pathogen acquisition and transmission. Rhipicephalus haemaphysaloides is one of the most widely distributed tick species in China. The symbiotic bacteria composition and their impacts to $R$. haemaphysaloides ticks have not been studied. The present study investigated the composition of microbial community in $R$. haemaphysaloides ticks and then assessed the effects of endosymbionts on the host's fecundity by antibiotic treatment experiments.

Methods: The microbial population of female and male $R$. haemaphysaloides ticks was analyzed using Illumina Miseq sequencing of 165 rRNA gene. Thirty engorged female ticks were then randomly divided into five groups and injected with ampicillin, ciprofloxacin, kanamycin, tetracycline, or phosphate-buffered solution (PBS), respectively. Effects of antibiotic treatments on maternal oviposition, egg hatching and density of endosymbionts were evaluated.
\end{abstract}

Results: Illumina Miseq sequencing showed that Coxiella and Rickettsia were the predominant bacterial genera inhabiting $R$. haemaphysaloides ticks. Antibiotic treatment experiments found that kanamycin reduced the density of Coxiella-like endosymbiont (Coxiella-LE hereafter) in eggs, ciprofloxacin reduced the density of Rickettsia-like endosymbiont (Rickettsia-LE), and tetracycline had effect on both endosymbionts, while ampicillin affected neither. Meanwhile hatching rates of eggs were observed to decrease greatly in the kanamycin or tetracycline-treated group but maintained in the ampicillin or ciprofloxacin-treated group. Furthermore, the reduced hatching rates were found to be associated with density of Coxiella-LE in eggs.

Conclusions: The findings indicate that Coxiella-LE is essential for the reproduction of $R$. haemaphysaloides ticks, and that kanamycin can be used to study the role of Coxiella-LE on ticks.

Keywords: Rhipicephalus haemaphysaloides, Endosymbiont, Coxiella, Rickettsia, Fecundity

\section{Background}

Endosymbiotic bacteria inhabit a variety of arthropods. Some bacteria exhibit multiple effects on the host's survival, reproduction or pathogen acquisition and transmission [1]. Ticks are obligate hematophagous arthropods and are considered second only to mosquitoes as vectors of human disease in the world [2]. To date, endosymbiotic

\footnotetext{
* Correspondence: zhudan818@126.com

${ }^{2}$ National Institute of Parasitic Diseases, Chinese Center for Disease Control and Prevention, WHO Collaborating Centre for Malaria, Schistosomiasis and Filariasis, Key Laboratory of Parasite \& Vector Biology, Ministry of Health, Shanghai 200025, People's Republic of China

Full list of author information is available at the end of the article
}

bacteria of the genera Coxiella, Rickettsia, Francisella, Wolbachia, Arsenophonus or "Candidatus Midichloria mitochondrii" have been found in ticks [3]. Some of them were found to have important roles in ticks' survival or reproduction. Zhong et al. [4] suggested that reproductive fitness of A. americanum was reduced after treated with rifampin or tetracycline, and reduction in fecundity was probably related to the density of Coxiella-like endosymbiont (Coxiella-LE). However, they could not rule out the contribution of the Rickettsia to the effects because the antibiotics used in the study (rifampin and tetracycline) could reduce the density of Coxiella-LE and Rickettsia-like 
endosymbiont (Rickettsia-LE) simultaneously. In other studies, Zhang et al. [5] found that reproductive fitness of $H$. longicornis decreased dramatically after the density of Coxiella-LE was reduced by tetracycline treatment, while Andre et al. [6] revealed that although the density of Rickettsia-LE decreased significantly after treatment with tetracycline or ciprofloxacin, it did not affect the fecundity of I. pacificus ticks. Meanwhile "Candidatus Midichloria mitochondrii" was found to have the ability to impact the process of engorgement and molt in I. ricinus ticks [7]. In addition, bacterial symbionts can affect pathogen acquisition and transmission of ticks. For example, disturbing microbiota by antibiotic treatment could affect the susceptibility of I. scapularis ticks to Borrelia burgdorferi, Anaplasma phagocytophilum, A. marginale and Francisella novicida [8-10]. However, the role of tick endosymbionts remains poorly understood because the existing studies are limited to a few tick species [11].

Rhipicephalus haemaphysaloides is widely distributed in China $[12,13]$. It has been reported to be vector of several pathogens including Kyasanur Forest disease virus, Babesia microti, Ehrlichia canis and so on $[14,15]$. In tick species of Rhipicephalus genus, bacterial communities in $R$. turanicus, $R$. sanguineus, $R$. annulatus and $R$. microplus have been analyzed [11, 16-18]. Coxiella, Rickettsia, Wolbachia and "Candidatus Midichloria mitochondrii" have been reported in Rhipicephalus ticks. However, the symbiotic bacterial composition of $R$. haemaphysaloides and their impacts to the hosts have not been studied. In order to test whether endosymbiont influence the fecundity of $R$. haemaphysaloides, we assessed the effects of endosymbionts on maternal oviposition and hatching of eggs by antibiotic treatment experiments.

\section{Methods}

\section{Ticks}

An engorged $R$. haemaphysaloides female tick was removed from a dog in Tengchong County, Yunnan Province of China. The colony was then maintained in the laboratory in an incubator at $25{ }^{\circ} \mathrm{C}$, with $85 \%$ relative humidity and a 14/10 h light/dark photoperiod regimen as described previously [14]. Adult ticks were fed on New Zealand white rabbit.

\section{Microbial population analysis}

The DNA of unfed female or male ticks was extracted using DNeasy Blood \& Tissue Kit (Qiagen, Hilden, Germany) according to the manufacturer's protocol.

DNA samples of 15 female or 15 male ticks were pooled together in equal concentrations. The microbial population of females and males were then analyzed separately using Illumina MiSeq sequencing by Shanghai Majorbio Bio-pharm Technology Company Limited (Shanghai, China). In brief, the V3-V4 variable region of the bacterial $16 S$ rRNA gene was amplified by PCR using the universal primers $338 \mathrm{~F}$ and $806 \mathrm{R}$ (Table 1) under conditions of $95{ }^{\circ} \mathrm{C}$ for $2 \mathrm{~min}$, followed by 25 cycles at $95{ }^{\circ} \mathrm{C}$ for $30 \mathrm{~s}, 55^{\circ} \mathrm{C}$ for $30 \mathrm{~s}$ and $72{ }^{\circ} \mathrm{C} 30 \mathrm{~s}$ with a final extension at $72{ }^{\circ} \mathrm{C}$ for $5 \mathrm{~min}$ [19]. PCR amplifications were performed in quintuplicate for each sample. Purified PCR products were pooled together in equal molar concentrations and sequenced by Illumina MiSeq platform according to standard protocols. Sequences with 97\% similarity were clustered into the same operational taxonomic units (OTUs), with one sequence per OTU being selected as a representative sequence for further downstream analysis [8]. For each representative sequence, the GreenGene Database was applied in annotating taxonomic information with RDP classifier (v.2.2). Alpha diversity was estimated using the ACE, Chao1, Shannon's and Simpson's indices.

\section{Antibiotic treatments}

In total, 30 engorged female ticks dropping from the rabbits were weighed and then randomly divided into five treatment groups: ampicillin, ciprofloxacin, kanamycin, tetracycline, and phosphate buffered solution (PBS), with six ticks in each group. Antibiotic solutions were made at concentrations of $10 \mathrm{mg} / \mathrm{ml}$. Each engorged tick was injected into the hemocoel between the first and second legs using microinjection needles. The doses of each solution used for injection were $1 \mu \mathrm{l}$ per $100 \mathrm{mg}$ body weight

Table 1 Primers for PCR amplification and GPCR reaction

\begin{tabular}{|c|c|c|c|c|}
\hline Organism & Target gene & Primer & Sequence $\left(5^{\prime}-3^{\prime}\right)$ & Reference \\
\hline \multirow[t]{2}{*}{ Bacteria } & \multirow[t]{2}{*}{165} & $338 f$ & ACTCCTRCGGGAGGCAGCAG & \multirow[t]{2}{*}{ [19] } \\
\hline & & $806 r$ & GGACTACCVGGGTATCTAAT & \\
\hline \multirow[t]{2}{*}{ R. haemaphysaloides } & \multirow[t]{2}{*}{ Actin gene } & Ractin-F & GTGCCCATCTACGAAGGTTAC & \multirow[t]{2}{*}{ This study } \\
\hline & & Ractin-R & CCATCTCCTGCTCGAAGTCC & \\
\hline \multirow[t]{2}{*}{ Rickettsia-like endosymbiont } & \multirow[t]{2}{*}{ Citrate synthase gene $(g / t A)$} & gltA-F & TCCTACATGCCGACCATGAG & \multirow[t]{2}{*}{ [20] } \\
\hline & & gltA-R & AAAGGGTTAGCTCCGGATGAG & \\
\hline \multirow[t]{2}{*}{ Coxiella-like endosymbiont } & \multirow[t]{2}{*}{165 rRNA gene } & L-CoxF & TGAGTGTTGACGTTACCCACAG & \multirow[t]{2}{*}{ This study } \\
\hline & & L-CoxR & GCATTTCACCGCTACACCG & \\
\hline
\end{tabular}


of ticks. The needle was left inside tick body for $30 \mathrm{~s}$ after injection and was then withdrawn slowly [4].

Each injected tick was maintained in separate containers and monitored for oviposition daily. The period between dropping from the rabbit and the beginning of oviposition was recorded as time to oviposition. Ticks dying before oviposition were excluded from the study. Egg masses of each tick were weighed after completion of oviposition. The ratio of two weights, the total weight of egg mass to the weight of the engorged female before oviposition, was recorded as the oviposition index. After that, 100 randomly selected eggs from each female were preserved in $75 \%$ ethanol for further DNA extraction and molecular analysis. Another 200 randomly selected eggs from each female were put into a new container to estimate hatching rate. The remaining eggs were left in the original containers. Hatching states of eggs were monitored daily. The incubation period for eggs was defined as time from deposition of the first egg to the occurrence of the first larvae. For each container with 200 eggs, the number of hatched larvae was counted under a dissecting microscope after being frozen, and the percentage of hatched larvae was defined as the hatching rate for eggs.

\section{Quantitative polymerase chain reaction (qPCR) assay}

DNA of eggs from each group was extracted using the method described above. Relative densities of Coxiella-LE and Rickettsia-LE in eggs were analyzed using the SYBR green qPCR approach. The 165 rRNA gene fragment of Coxiella-LE, citrate synthase (gltA) gene fragment of Rickettsia-LE and the beta-actin biosynthetic (actin) gene fragment of $R$. haemaphysaloides ticks were qPCR-amplified (Table 1). The relative density of Coxiella-LE or Rickettsia-LE was defined as Coxiella $16 \mathrm{~S}$ rRNA or Rickettsia gltA gene copies per tick actin gene copy [20]. Specificity of all the primers was verified by PCR amplification and then sequencing of the PCR products. To further confirm the prevalence of Coxiella-LE and Rickettsia-LE in adult ticks, DNA samples of adult ticks were also analyzed separately by qPCR.

All qPCR reactions were performed in CFX96 RealTime PCR System (Bio-Rad laboratories Incorporation, Richmond, CA, USA). Each $15 \mu$ l reaction mixture contained 1.5 $\mu$ l DNA sample, $7.5 \mu \mathrm{l}$ qPCR Master Mix Plus (Takara Bio Inc, Shiga, Japan), $1 \mu \mathrm{l}$ of $5 \mathrm{mM}$ primers and $5 \mu \mathrm{l}$ of water. Four negative controls were used in each 96-well qPCR plate to exclude contamination. The cycling condition for each reaction was $95{ }^{\circ} \mathrm{C}$ for $30 \mathrm{~s}, 40$ cycles of $95^{\circ} \mathrm{C}$ for $5 \mathrm{~s}$ and $60{ }^{\circ} \mathrm{C}$ for $30 \mathrm{~s}$, with the fluorescence recorded at the annealing stage.

\section{Statistical analysis}

Statistical analysis was carried out with SPSS 19.0 software (IBM, Armonk, NY, USA). The relative density of Coxiella-LE and Rickettsia-LE was log-transformed for significance testing. One-way ANOVA was used to compare weight of ticks, oviposition index, log-transformed relative density of Coxiella-LE or Rickettsia-LE and hatching rate among groups. Difference between any of the antibiotic-treated group and PBS-treated group was analyzed by Dunnett's test. Time to oviposition and incubation period of eggs among groups were compared with the Kruskal-Wallis H-test, and Dunn's test was used to analyze the difference between antibiotic-treated and PBS-treated groups. Spearman's rank correlation and multiple linear regression model were used to analyze the association between relative density of Coxiella-LE or Rickettsia-LE and incubation period or hatching rate for eggs. Significant difference was defined as $P<0.05$ with a two-tailed test.

\section{Results}

Microbial community composition in unfed $R$. haemaphysaloides adult ticks

In total, 38,599 sequence reads were obtained from females and 41,109 reads from males of $R$. haemaphysaloides after trimming and removing all low-quality sequences. Twenty-four different bacterial genera were identified from female ticks and 57 genera were identified from males. Among all the bacterial genera, 21 were shared by both females and males. Bacterial genera with relative abundance higher than $1 \%$ in female or male ticks including Coxiella, Rickettsia, Mycobacterium, Brevibacterium, Pseudomonas, Acinetobacter, Dietzia and Staphylococcus. Coxiella and Rickettsia were the predominant genera (Fig. 1). The results of qPCR further revealed that prevalences of both Coxiella-LE and Rickettsia-LE were $100 \%$ in adult ticks. Except for Coxiella and Rickettsia, all the above genera were extracellular bacteria and were probably derived from the guts of ticks or the environment.

Alpha diversity of female and male ticks is shown in Table 2. Community richness revealed by ACE and

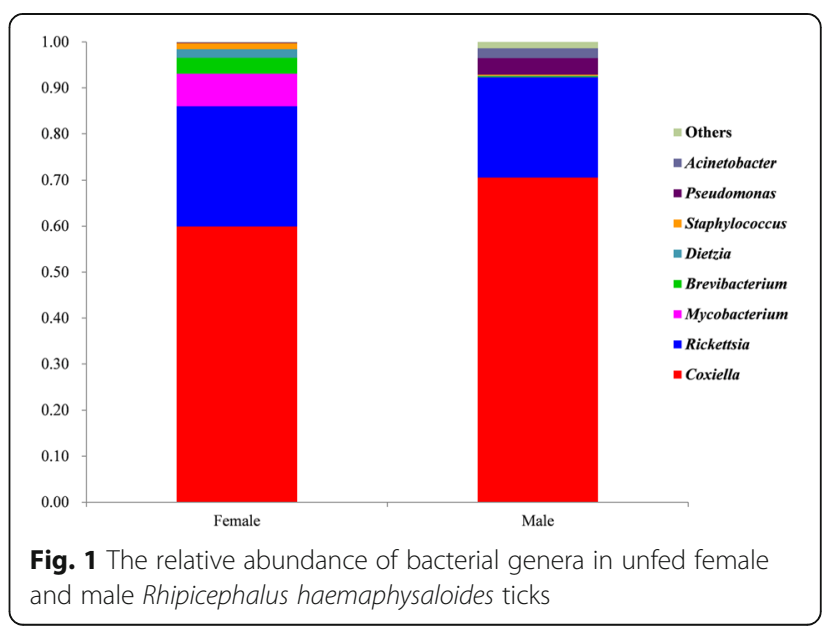


Table 2 Alpha-diversity (confidence interval) of female and male R. haemaphysaloides ticks

\begin{tabular}{llllll}
\hline Ticks & ACE estimator & Chao1 estimator & Simpson's index & Shannon's index & Coverage (\%) \\
\hline Female & $37.47(33.45-52.67)$ & $35.50(32.65-50.89)$ & $0.43(0.43-0.44)$ & $1.13(1.12-1.14)$ & 99.98 \\
Male & $75.08(71.58-86.40)$ & $72.77(70.56-83.60)$ & $0.55(0.54-0.55)$ & $0.93(0.92-0.94)$ & 99.98
\end{tabular}

Chaol estimators was higher in male ticks than that in females; however, community diversity estimated by Shannon's and Simpson's indices was higher in females than that in males.

\section{Effects of antibiotic treatment on tick fecundity and density of Coxiella-LE and Rickettsia-LE}

One tick from the tetracycline group died without laying eggs and was excluded from data analysis. The average weight of engorged ticks among five groups was not statistically different (data not shown).

Indicators of the fecundity and relative density of Coxiella-LE and Rickettsia-LE in eggs from each group are summarized in Table 3 and Figs. 2, 3, 4, 5, 6 and 7.

When compared with PBS-treated group, time to oviposition was delayed in tetracycline-treated group (Dunn's test: $Z=18.98, P=0.002$ ), but it was not significantly changed in ampicillin-, ciprofloxacinand kanamycin-treated groups (Fig. 2). Oviposition index and incubation period of eggs were not statistically different among five treatment groups (Figs. 3 and 4). However, hatching rates of eggs in the kanamycin-treated group (15.3\%) and tetracyclinetreated group (5.6\%) were significantly lower than that in the PBS- (94.7\%), ampicillin- (96.5\%) or ciprofloxacin-treated (94.5\%) groups (Table 3 and Fig. 5).

As shown in Fig. 6, the estimated relative density of Coxiella-LE in eggs of both the tetracycline- and kanamycin-

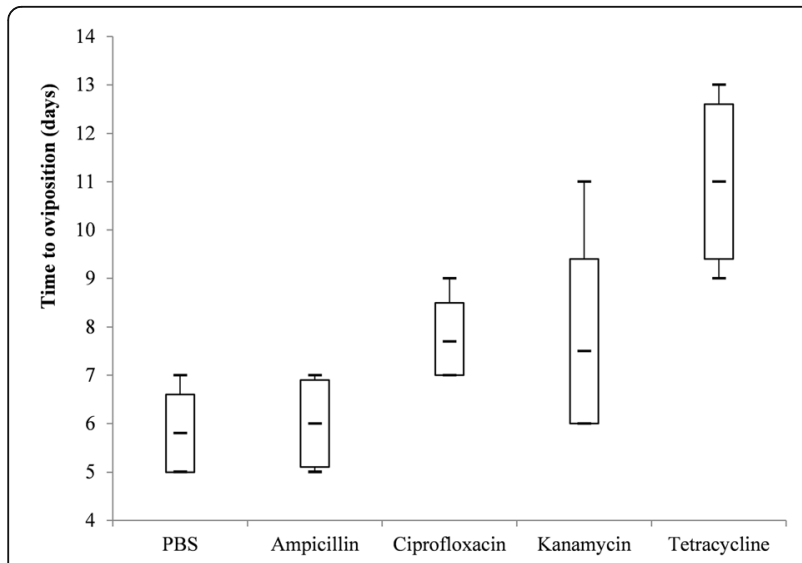

Fig. 2 Box-and-whisker plots of the time to oviposition in five treatment groups of $R$. haemaphysaloides treated ticks were lower than that of PBS-treated ticks, while it was not statistically different between ampicillinor ciprofloxacin-treated groups and PBS-treated groups. Meanwhile, the relative density of Rickettsia-LE in eggs was reduced by ciprofloxacin or tetracycline treatment, but not affected by kanamycin or ampicillin injection when comparing to PBS treatment (Fig. 7).

\section{Association between density of Coxiella-LE or Rickettsia-LE and egg hatching}

As shown in Table 4, the hatching rate of eggs was positively correlated with the relative density of both Coxiella-LE and Rickettsia-LE in eggs (Spearman's correlation coefficient $=0.89, P<0.001$ for CoxiellaLE; Spearman's correlation coefficient $=0.57, P<0$. 001 for Rickettsia-LE). Multiple linear regression analysis was conducted to analyze the separate effects of Coxiella-LE and Rickettsia-LE. It revealed that only the density of Coxiella-LE was associated with hatching rate, but there was no association for RickettsiaLE (Table 5).

\section{Discussion}

The microbiome of $R$. haemaphysaloides ticks has not been studied previously. In the present study, microbial communities of adult $R$. haemaphysaloides ticks were analyzed based on the Illumina Miseq sequencing of $16 S$ rRNA gene. Consistent with the results in $R$. sanguineus and $R$. turanicus ticks $[16,17]$, our study revealed that Coxiella and Rickettsia were

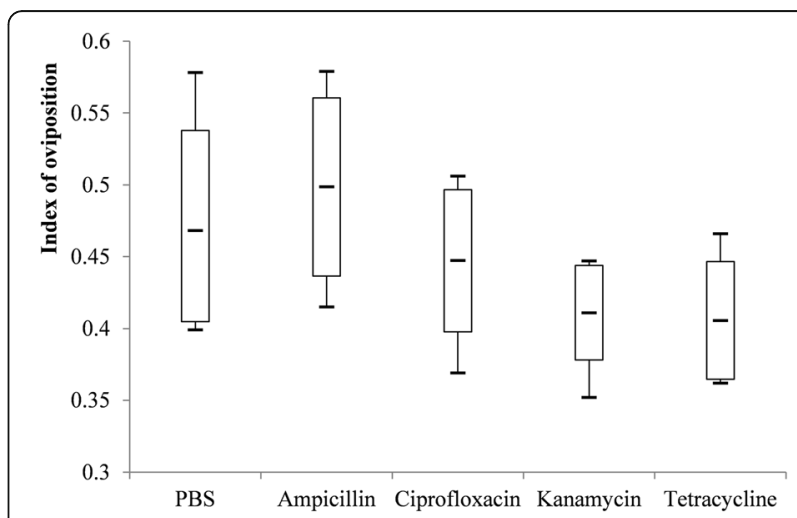

Fig. 3 Box-and-whisker plots of the index of oviposition in five treatment groups of $R$. haemaphysaloides 


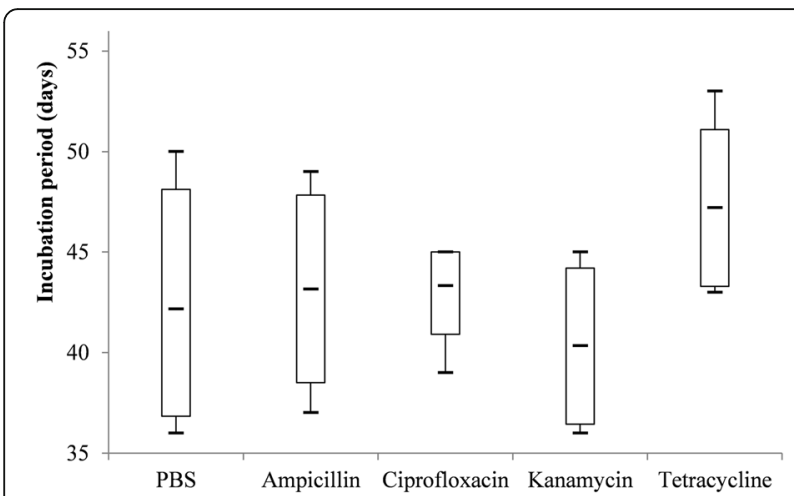

Fig. 4 Box-and-whisker plots of the incubation period of eggs in five treatment groups of $R$. haemaphysaloides

the predominant genera of both female and male $R$. haemaphysaloides ticks. However, in $R$. annulatus and $R$. microplus ticks, neither Coxiella nor Rickettsia was the predominant symbiotic bacteria [18, 21]. Interestingly, within Rhipicephalus, both $R$. annulatus and $R$. microplus belong to the subgenus of Boophilus, while $R$. sanguineus, $R$. turanicus and $R$. haemaphysaloides belong to another phylogenic clade [22]. Therefore, the differences of microbiome among tick species may reflect the co-evolution of symbionts with host. It can be argued that wild collected $R$. haemaphysaloides ticks may have a different microbiome composition with laboratory reared ticks. However, it is rational to speculate that Coxiella and Rickettsia are also the predominant genera for wild collected ticks because both Coxiella-LE and Rickettsia-LE are transovarially transmitted while extracellular bacteria are often obtained from the environment [23]. The speculation was also confirmed by microbial community analysis of 13 engorged female $R$. haemaphysaloides ticks removed from

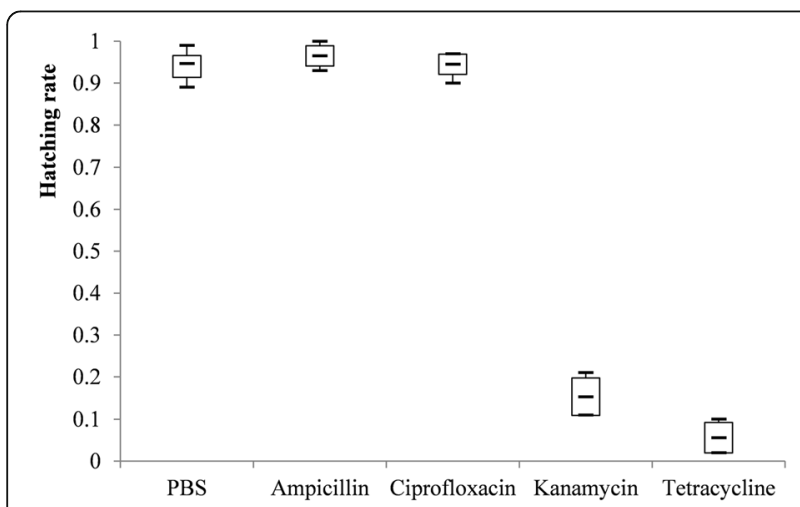

Fig. 5 Box-and-whisker plots of the hatching rate of eggs in five treatment groups of $R$. haemaphysaloides

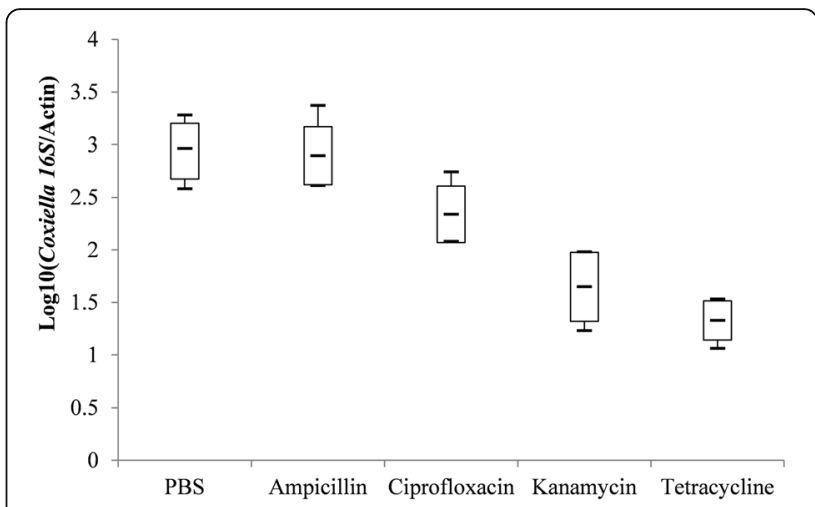

Fig. 6 Box-and-whisker plots of log-transformed relative density of Coxiella-like endosymbiont in five treatment groups of R. haemaphysaloides

domestic animals in Tengchong County. The results showed that Coxiella-LE, Rickettsia-LE and pathogenic Anaplasma were the most predominant bacteria in the field collected samples, with relative abundances of $68.5,12.6$ and $26.4 \%$, respectively (data not shown).

The difference of microbial diversity between female and male ticks was studied previously in Dermacentor occidentalis, I. scapularis and A. americanum ticks [24]. The results showed that female ticks harbored a less diverse array of bacteria than males. The authors supposed that higher abundance of Rickettsia-LE and Francisella endosymbionts led to lower community diversity in female ticks than males because endosymbionts were thought to partially exclude the invasion of other bacteria. Unlike the previous study, we found that community richness was higher in male ticks than females, while

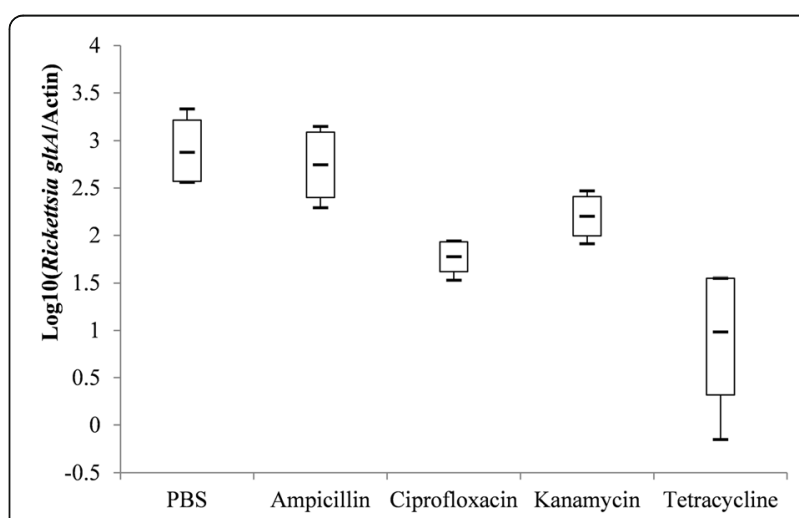

Fig. 7 Box-and-whisker plots of log-transformed relative density of Rickettsia-like endosymbiont in five treatment groups of R. haemaphysaloides 
Table 3 Effects of antibiotic treatment on fecundity R. haemaphysaloides and density of Coxiella-LE and Rickettsia-LE in eggs

\begin{tabular}{llllllll}
\hline Treatment & $\begin{array}{l}\text { No. of } \\
\text { Ticks }\end{array}$ & $\begin{array}{l}\text { Time to } \\
\text { oviposition } \\
\text { (days) }\end{array}$ & $\begin{array}{l}\text { Index of } \\
\text { oviposition }\end{array}$ & $\begin{array}{l}\text { Incubation } \\
\text { period (days) }\end{array}$ & Hatching rate & $\begin{array}{l}\text { Log-transformed } \\
\text { relative density } \\
\text { of Coxiella-LE }\end{array}$ & $\begin{array}{l}\text { Log-transformed } \\
\text { relative density of } \\
\text { Rickettsia-LE }\end{array}$ \\
\hline PBS & 6 & $5.8 \pm 0.8$ & $0.5 \pm 0.06$ & $42.2 \pm 5.4$ & $0.95 \pm 0.03$ & $3.0 \pm 0.3$ & $2.9 \pm 0.3$ \\
Ampicillin & 6 & $6.0 \pm 0.9$ & $0.5 \pm 0.05$ & $43.2 \pm 4.7$ & $0.97 \pm 0.02$ & $2.9 \pm 0.3$ & $2.8 \pm 0.4$ \\
Ciprofloxacin & 6 & $7.7 \pm 0.8$ & $0.4 \pm 0.03$ & $43.3 \pm 2.4$ & $0.95 \pm 0.02$ & $2.3 \pm 0.3$ & $1.8 \pm 0.2^{\mathrm{a}}$ \\
Kanamycin & 6 & $7.5 \pm 1.9$ & $0.5 \pm 0.06$ & $40.3 \pm 3.9$ & $0.15 \pm 0.04^{\mathrm{a}}$ & $1.7 \pm 0.3^{\mathrm{a}}$ & $2.2 \pm 0.2$ \\
Tetracycline & 5 & $11.0 \pm 1.6^{\mathrm{a}}$ & $0.4 \pm 0.04$ & $47.2 \pm 3.9$ & $0.06 \pm 0.04^{\mathrm{a}}$ & $1.3 \pm 0.2^{\mathrm{a}}$ & $1.0 \pm 0.7^{\mathrm{a}}$ \\
F-value & & - & 3.30 & - & 307.8 & 39.4 & 24.6 \\
$P$-value & $<0.01^{\mathrm{b}}$ & 0.03 & $0.16^{\mathrm{c}}$ & $<0.01$ & $<0.01$ & $<0.01$ \\
\hline
\end{tabular}

${ }^{a}$ The value is statistically different from that of PBS-treated group

${ }^{b}$ Kruskal-Wallis H-test: $\chi^{2}=16.459, d f=4$

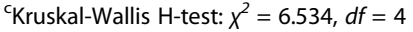

community diversity was higher in females than males (Table 2). However, the biological significance of these differences still needs to be investigated.

To date, a limited number of studies have been conducted to investigate the effects of endosymbionts on fecundity of ticks. In the present study, engorged female $R$. haemaphysaloides ticks were treated with one of the five solutions including ampicillin, ciprofloxacin, kanamycin, tetracycline and PBS. The results showed that kanamycin reduced the density of Coxiella-LE in eggs and ciprofloxacin reduced the density of Rickettsia-LE, while tetracycline had effect on both endosymbionts and ampicillin had effect on neither (Table 2). Moreover, the hatching rates of eggs were observed to decrease greatly in the kanamycin- or tetracycline-treated groups but it maintained in the ciprofloxacin- or ampicillin-treated groups. Therefore, we suggested that Coxiella-LE was related to the reduced hatching rates of eggs, and that it was a primary endosymbiont of $R$. haemaphysaloides. Meanwhile, Rickettsia-LE was probably a secondary endosymbiont of ticks [6]. Consistent with the previous study, time to oviposition was delayed in the tetracycline-treated group [4, 6]. It was supposed that delaying oviposition in ticks by tetracycline treatment should lie in reasons other than the clearance of Coxiella-LE, because delaying oviposition was not observed in ticks treated with kanamycin. Thus we suspected tetracycline might influence ticks

Table 4 Spearman's correlation coefficient between relative density of Coxiella-LE or Rickettsia-LE and egg hatching of $R$. haemaphysaloides

\begin{tabular}{llll}
\hline Variables & $\begin{array}{l}\text { Incubation } \\
\text { period }\end{array}$ & $\begin{array}{l}\text { Hatching } \\
\text { rate }\end{array}$ & Log(Cox16S/Actin) \\
\hline Log(Ric-gltA/Actin) & -0.27 & $0.57^{*}$ & $0.76^{*}$ \\
Log(Cox16S/Actin) & -0.20 & $0.89^{*}$ & - \\
\hline
\end{tabular}

${ }^{*} P<0.001$ by certain unknown mechanism, and kanamycin was considered to be the better choice to disturb Coxiella-LE than tetracycline in related studies. In addition, since ampicillin does not have an effect on either of the two endosymbionts, it can be used to study the function of gut microbiome in $R$. haemaphysaloides ticks. Distinct from the study on A. americanum ticks [4], the index of oviposition and the incubation period of larvae were not significantly altered by any of the antibiotics. The reason might lie in the relatively limited number of ticks used in our study. The present study can be challenged that it cannot exclude completely the impact of symbiotic bacteria other than Coxiella-LE and Rickettsia-LE on the reproduction. However, considering their vertical transmitted feature, endosymbionts are more probable to manipulate reproduction of ticks than extracellular bacterial symbionts.

\section{Conclusions}

Our study found that $R$. haemaphysaloides ticks harbored two endosymbionts, namely Coxiella-like and Rickettsialike endosymbionts; the Coxiella-like endosymbiont was considered to be related to fecundity of $R$. haemaphysaloides ticks and kanamycin was considered to be suitable antibiotic to manipulate the density of Coxiella-like endosymbiont.

Table 5 The influence of Coxiella-LE and Rickettsia-LE on hatching rate of $R$. haemaphysaloides in multiple linear regression model

\begin{tabular}{llllll}
\hline Variables & Coefficient & $\begin{array}{l}\text { Standard } \\
\text { error }\end{array}$ & $\begin{array}{l}\text { Standard } \\
\text { coefficient }\end{array}$ & $t$-value & $P$-value \\
\hline Intercept & -0.53 & 0.12 & & -4.4 & $<0.001$ \\
Log & -0.14 & 0.07 & -0.26 & -2.0 & 0.06 \\
$\begin{array}{l}\text { (Ric-gltA/Actin) } \\
\text { Log }\end{array}$ & 0.65 & 0.08 & 1.08 & 8.3 & $<0.001$ \\
\begin{tabular}{l} 
(Cox16S/Actin) \\
\hline
\end{tabular} & & & & & \\
\hline
\end{tabular}




\section{Abbreviations}

Coxiella-LE: Coxiella-like endosymbiont; gltA: citrate synthase gene; OTU: operational taxonomic unit; PBS: phosphate buffer saline; RDP: Ribosomal Database Project; Rickettsia-LE: Rickettsia-like endosymbiont

\section{Acknowledgements}

We thank Dr Yan Lu and Mrs Yu-Chun Cai for the help with tick maintenance in the laboratory.

\section{Funding}

The research has been partially supported by National Key Research and Development Program of China (Nos. 2016YFC1202001 and 2016YFC1200500), the fourth round of Three-Year Public Health Action Plan (2015-2017, No. GWIV-29), the Special Fund for Health Research in the Public Interest China (No. 201202019), and the Scientific Research Foundation for Doctors of Weifang Medical College (No. 2017BSQD52).

\section{Availability of data and materials}

The sequencing data used in this article have been submitted to the National Center for Biotechnology Information's Sequence Read Archive (accession Nos. SAMN08715268 and SAMN08715268).

\section{Authors' contributions}

LHL designed and performed the experiments and drafted the manuscript. $D Z$ and $Y Z$ conceived the study and revised the manuscript. All authors read and approved the final manuscript.

\section{Ethics approval and consent to participate}

All procedures for animal experiments were approved by the Ethics Committee at the National Institute of Parasitic Diseases (NIPD), Chinese Center for Disease Control and Prevention (IPD-2016-12, June 30, 2016).

\section{Consent for publication}

Not applicable.

\section{Competing interests}

The authors declare that they have no competing interests.

\section{Publisher's Note}

Springer Nature remains neutral with regard to jurisdictional claims in published maps and institutional affiliations.

\footnotetext{
Author details

${ }^{1}$ School of Publish Health and Management, Weifang Medical University, Weifang 261053, People's Republic of China. ${ }^{2}$ National Institute of Parasitic Diseases, Chinese Center for Disease Control and Prevention, WHO Collaborating Centre for Malaria, Schistosomiasis and Filariasis, Key Laboratory of Parasite \& Vector Biology, Ministry of Health, Shanghai 200025, People's Republic of China.
}

Received: 27 December 2017 Accepted: 22 March 2018

Published online: 13 April 2018

\section{References}

1. Dale C, Moran NA. Molecular interactions between bacterial symbionts and their hosts. Cell. 2006;126:453-65.

2. Fang LQ, Liu K, Li XL, Liang S, Yang Y, Yao HW, et al. Emerging tick-borne infections in mainland China: an increasing public health threat. Lancet Infect Dis. 2015;15:1467-79.

3. Liu LM, Liu JN, Liu Z, Yu ZJ, Xu SQ, Yang XH, et al. Microbial communities and symbionts in the hard tick Haemaphysalis longicornis (Acari: Ixodidae) from north China. Parasit Vectors. 2013:6:310.

4. Zhong J, Jasinskas A, Barbour AG. Antibiotic treatment of the tick vector Amblyomma americanum reduced reproductive fitness. PLoS One. 2007;2:e405.

5. Zhang CM, Li NX, Zhang TT, Qiu ZX, Li Y, Li LW, Liu JZ. Endosymbiont CLS-HI plays a role in reproduction and development of Haemaphysalis longicornis. Exp Appl Acarol. 2017;73(3-4):429-38.

6. Kurlovs AH, Li J, Cheng D, Zhong J. Ixodes pacificus ticks maintain embryogenesis and egg hatching after antibiotic treatment of Rickettsia endosymbiont. PLoS One. 2014;9:e104815.
7. Ninio C, Plantard O, Serra V, Pollera C, Ferrari N, Cafiso A, et al. Antibiotic treatment of the hard tick Ixodes ricinus: influence on Midichloria mitochondrii load following blood meal. Ticks Tick Borne Dis. 2015;6:653-7.

8. Abraham NM, Liu L, Jutras BL, Yadav AK, Narasimhan S, Gopalakrishnan V, et al. Pathogen-mediated manipulation of arthropod microbiota to promote infection. Proc Natl Acad Sci USA. 2017;114:e781-90.

9. Narasimhan S, Rajeevan N, Liu L, Zhao YO, Heisig J, Pan J, et al. Gut microbiota of the tick vector Ixodes scapularis modulate colonization of the Lyme disease spirochete. Cell Host Microbe. 2014;15:58-71.

10. Gall CA, Reif KE, Scoles GA, Mason KL, Mousel M, Noh SM, Brayton KA. The bacterial microbiome of Dermacentor andersoni ticks influences pathogen susceptibility. ISME J. 2016;10:1-10.

11. Ahantarig A, Trinachartvanit W, Baimai V, Grubhoffer L. Hard ticks and their bacterial endosymbionts (or would be pathogens). Folia Microbiol. 2013;58:419-28.

12. Yu Z, Wang H, Wang T, Sun W, Yang X, Liu J. Tick-borne pathogens and the vector potential of ticks in China. Parasit Vectors. 2015:8:24.

13. Chen Z, Yang X, Bu F, Yang X, Yang X, Liu J. Ticks (Acari: Ixodoidea: Argasidae, Ixodidae) of China. Exp Appl Acarol. 2010;51:393-404.

14. Li LH, Zhu D, Zhang CC, Zhang Y, Zhou XN. Experimental transmission of Babesia microti by Rhipicephalus haemaphysaloides. Parasit Vectors. 2016;9:231.

15. Zhang J, Liu Q, Wang D, Li W, Beugnet F, Zhou J. Epidemiological survey of ticks and tick-borne pathogens in pet dogs in south-eastern China. Parasite. 2017;24:35.

16. Lalzar I, Harrus S, Mumcuoglu KY, Gottlieb Y. Composition and seasonal variation of Rhipicephalus turanicus and Rhipicephalus sanguineus bacterial communities. Appl Environ Microbiol. 2012;78:4110-6.

17. Rene-Martellet M, Minard G, Massot R, Tran W, Valiente MC, Chabanne L, Mavingui P. Bacterial microbiota associated with Rhipicephalus sanguineus (s.l.) ticks from France, Senegal and Arizona. Parasit Vectors. 2017:10:416.

18. Tekin S, Dowd SE, Davinic M, Bursali A, Keskin A. Pyrosequencing based assessment of bacterial diversity in Turkish Rhipicephalus annulatus and Dermacentor marginatus ticks (Acari: Ixodidae). Parasitol Res. 2017;116:1055-61.

19. Liu JH, Zhang ML, Zhang RY, Zhu WY, Mao SY. Comparative studies of the composition of bacterial microbiota associated with the ruminal content, ruminal epithelium and in the faeces of lactating dairy cows. Microb Biotechnol. 2016:9:257-68.

20 Jasinskas A, Zhong J, Barbour AG. Highly prevalent Coxiella sp. bacterium in the tick vector Amblyomma americanum. Appl Environ Microbiol. 2007;73:334-6.

21 Andreotti R, Perez AA, Dowd SE, Guerrero FD, Bendele KG, Scoles GA. Assessment of bacterial diversity in the cattle tick Rhipicephalus (Boophilus) microplus through tag-encoded pyrosequencing. BMC Microbiol. 2011;11:6

22 Nava S, Guglielmone AA, Mangold AJ. An overview of systematics and evolution of ticks. Front Biosci. 2009;14:2857-77.

23 Kwan JY, Griggs R, Chicana B, Miller C, Swei A. Vertical vs. horizonta transmission of the microbiome in a key disease vector, Ixodes pacificus. Mol Ecol. 2017:26:6578-89.

24 Gurfield N, Grewal S, Cua LS, Torres PJ, Kelley ST. Endosymbiont interference and microbial diversity of the Pacific coast tick, Dermacentor occidentalis, in San Diego County, California. PeerJ. 2017;5:e3202.

\section{Submit your next manuscript to BioMed Central and we will help you at every step:}

- We accept pre-submission inquiries

- Our selector tool helps you to find the most relevant journal

- We provide round the clock customer support

- Convenient online submission

- Thorough peer review

- Inclusion in PubMed and all major indexing services

- Maximum visibility for your research

Submit your manuscript at www.biomedcentral.com/submit
Biomed Central 